\title{
The Effect of Action Observation Training on Sit to Walk with Chronic Stroke Patients
}

\author{
Jin-Cheol Kim¹, Hyun-Min Lee ${ }^{2}$ \\ ${ }^{1}$ Major in Physical Therapy, Department of Rehabilitation Science, Graduated School of Honam University, Gwangju; ${ }^{2}$ Department of Physical \\ Therapy, College of Health Science, Honam University, Gwangju, Korea
}

Purpose: This study was performed to examine the effect of action observation training on sit to-walk times in chronic stroke patients. Methods: Twelve stroke patients were randomly allocated to two groups, as follows: an action observation training (AOT) group and a scenery picture observation (SPO) group. The AOT group was engaged in video observation for 2 minutes 30 seconds with 12 ADL functional activities related to the sit-to-walk task: physical training was carried out in two repetitions lasting 2 minutes 30 seconds. The landscape imagery control group observed landscape picture for 2 minutes 30 seconds and then carried out the same physical training. The intervention involved a total of 12 sessions over 4 weeks, and each session lasted 30 minutes. The pre and post-tests measured the timed up and go test (TUG), Sit to stand test (STS), and Dynamic gait index (DGI). The Wilcoxon signed ranks test was used to compare pre-test and post-test result, and the Mann-Whitney $\mathrm{U}$ test was employed for comparison between groups.

Results: The TUG time was significantly different between the AOT group and the SPO group. According to the findings, the experimental group's TUG was significantly decreased $(p<0.05)$. In comparison of the two groups, there was no significant in STS or DGI between the AOT group and SPO group.

Conclusion: This study showed that the intervention can be used to decrease TUG time. Our research suggests that action observation training has a positive effect on sit-to-walk times in patients with chronic stroke.

Keywords: Action observation training, Sit to walk, Stroke

\section{INTRODUCTION}

Many people affected by strokes have sequelae such as hemiplegia or quadriplegia with mental, perceptual, motor, sensory or linguistic problems. ${ }^{1}$ Stroke patients mainly perform functional and daily living activities, using their unaffected limbs. They do not try to use their affected limbs. This physical inactivity leads to the learned non-use syndrome. ${ }^{2}$ Accordingly, they select asymmetric movement strategies and show compensatory actions in order to compensate for their reduced capacity. ${ }^{3}$

The reduced motor capacity after stroke limits the ability to perform functional activities required in daily life such as walking, stair climbing, the sit to stand (STS) movement, and the timed up

Received Nov 25, 2015 Revised Dec 22, 2015

Accepted Dec 24, 2015

Corresponding author Hyun-Min Lee

E-mail leehm@honam.ac.kr and go (TUG) movement. ${ }^{4}$ Stroke patients' movement capability and walking can start in a variety of positions. The TUG movement is an important factor in daily life as one of general functional activities. ${ }^{5}$ Senior citizens have difficulty in performing functional activities such as the STS movement. The STS is regarded as a major constraint in performing daily living activities. The movement of sitting and standing up from a lying down position requires the capability to maintain balance after standing up as a movement endpoint. ${ }^{6}$ However, the activity of standing up from a chair and walking is performed in one or more steps in daily life. For this reason, the TUG movement is regarded as a functional activity more important than the STS movement. ${ }^{7-9}$

The start of the STS movement and ambulation is related to the

Copylight (C) 2015 The Korea Society of Physical Therapy

This is an Open Access article distribute under the terms of the Creative Commons Attribution Non-commercial License (Http:// creativecommons.org/license/by-nc/3.0.) which permits unrestricted non-commercial use, distribution, and reproduction in any medium, provided the original work is properly cited. 
capability to generate the appropriate force so that the amount of initial load applied to the lower limb can push forward the body. ${ }^{10} \mathrm{~A}$ difference between the STS and the TUG movement have been studied from various perspectives. The ground reaction force of the feet, coordination movements, and the transfer of momentum are important at the moment that the hips are lifted from a sitting position. ${ }^{7}$ Like the STS movement, the positions of the feet, the height of a chair, armrests, speed, muscular strength, and other become important factors. ${ }^{11}$ Moreover, when stroke patients do the STS movement, kinematic energy reduces and the weight distribution becomes asymmetric. ${ }^{12}$ The largest difference between the STS and the TUG movement is that the TUG movement includes the start of ambulation. The TUG movement is composed of two movement tasks. When standing up from a sitting position, a person starts walking before stretching and extending his or her body.

It takes stroke patients more time 25 to $61 \%$ more time to stand up from a chair than healthy people. ${ }^{12}$ They also put more weight on the unaffected leg. Therefore, the vertical load applied to the affected leg reduces by about $20-25 \%$ while performing this task. ${ }^{11,13}$ Refshange et al. ${ }^{14}$ discovered that most stroke patients had difficulty in doing the TUG movement in a row. They identified that they used a non-fluid strategy that they started walking after fully standing up. ${ }^{14,15}$ Moreover, it took them 1.6 times more time to do the TUG movement than normal people. ${ }^{14}$

Many studies have reported that task-specific training is more effective than traditional approaches in order to improve stroke patients' capability in performing functional activities. ${ }^{16}$ The task-specific training is to practice the tasks suitable for patients' capabilities repeatedly. However, patients who have poor remaining capacity or do not receive the input related to the experience-dependent neuronal reversibility. ${ }^{17}$ The way to overcome this limit is the movement observation training currently proposed for neurological rehabilitation. It is a therapeutic approach based on the mirror neuron system, its neurological mechanism. ${ }^{18}$

In a study conducted by Fogassi (2005) et al. ${ }^{19}$ the mirror neuron system is more activated when goal-oriented movements rather than simple movements, or object-related movements rather than imitating movements are observed. Moreover, it is much more activated when the images of moving objects rather than those of stationary objects are observed. ${ }^{20}$ The human mirror neuron system is considered to be involved in imitation and learning. ${ }^{21,22}$ Based on these facts, there have recently been many studies intended to apply the movement observation and physical training to neurological rehabilitation programs.

There have also been many studies on the movement observation and physical training, and the movement observation training related to ambulatory training and daily living movements. ${ }^{23} \mathrm{Howev}$ er, there is a lack of studies on the STS and the TUG movement, functional activities, among ambulatory capabilities. In this context, this study aimed to observe the movement of TUG, a functional activity in daily life and the effects of movement observation training on chronic stroke patients' TUG movements.

\section{METHODS}

\section{Subjects}

This study intended for twelve chronic stroke patients who had received inpatient treatments in C Hospital in Gwangju. They fully understood the content of this study and agreed to participate in experiments. Ethical approval was obtained to commence this study from the Biomedical Ethics Committee of Honam University. The approval number was 10412223-201405-HR-010-01. For inclusion in this study, subjects had to meet the following criteria: six months or more had passed after a stroke was diagnose; there was no sign of cognitive impairment, visual and auditory disorders, and orthopedic disorders on the lower on the lower extremity; a person was able to sit and stand up independently; and a person was able to walk more than $10 \mathrm{~m}$, with or without an assistant device.

Twelve stroke patients were randomly divided into two groups, a scenery picture observation (SPO) group and an action observation training (AOT) group. One patient dropped out of the SPO group in the study process due to his/her hospital discharge. Two group members had received training three times a week, a total of twelve times for four weeks. Subjects' movements were recorded with a video camera to examine the pre-training functional evaluation tools such as the TUG test, the Dynamic Gait Index (DGI), and the STS test as well as the displacement of individuals' TUG movements. An evaluation was made in the same way after four-week training. The members of the SPO group $(n=5)$ observed static scenery pictures related to mountains, beaches, and rural areas, while those of the AOT group $(n=6)$ made an observation on a video tape composed of the STS and the TUG movement. 


\section{Experimental methods}

1) Measurement

(1) STS test

The STS movement was measured with a STS test. The instruction for this test was explained in a state that a subject leaned back in a 43 cm high chair with a backrest: "Quickly repeat standing up from a sitting position and sitting in a chair five times." A subject started his or her movement according to the verbal instruction of "Start", and ended them when his or her hips hit the bottom of the chair seat fifthly. The time consumed was recorded.

\section{(2) Dynamic Gait Index}

The DGI is a tool used to evaluate the balance and ambulation of people or senior citizens who have disorders in balance and the vestibular system, much required in daily life. It is a standardized evaluation tool to evaluate changes in ambulation which responds to ambulatory tasks. In this study, it is composed of eight ambulatory tasks: walking on a flat floor, walking by changing the speed, walking by turning the head vertically or horizontally, walking by crossing obstacles or around obstacles, walking up and down the stairs, and others. The grade between 0 and 3 was assigned according to the level of performance. The scores ranged from 0 to 24 , with a perfect score of 24 points. It was interpreted that the relevant subject was able to move safely when the score was 22 or more, whereas he or she might have a risk of falling when it was below 19.

\section{(3) Timed Up and Go test}

The TUG test is a test designed to evaluate a person's functional mobility, movement capability, and dynamic balance control. It is used to evaluate senior citizens' balance control and functional mobility to predict their risk of falling. In this study, a subject leaned back in a chair with $56 \mathrm{~cm}$ high armrests and placed his or her arms on the armrests. According to the sound of "Start", he or she stood up, walked $3 \mathrm{~m}$ along a line on the floor at a comfortable and safe speed, walked back to the chair, and sat down in the chair. An observer measured to record the time period from the moment that the sound of "Start" is made to the time that the subject sits down in the chair. Moreover, the tasks such as walking with a cup of water or counting numbers backwards were added.

\section{Intervention}

The AOT group and the SPO group selected two pictures and videos a day and observed them for 30 minutes. Each subject watched them from the laptop prepared in the therapy room. The SPO group watched static scenery pictures related to mountains, beaches, and rural areas for 2 minutes and 30 seconds, and did STS and TUG movements for 12 minutes and 30 seconds. On the other hand, the AOT group repeatedly practiced observed movements for 12 minutes, and trained them twice for 15 minutes each in the therapy room and 30 seconds after watching the video related to the STS and the TUG movement for 2 minutes and 30 seconds.

Ten tasks often used in daily life were presented as follows: 1) sit at a Bobath table, stand up, and walk; 2) sit in a therapy bed, stand up, and walk; 3) sit in a chair with wheels, stand up, and walk; 4) sit in a chair with handles, stand up, and walk 5) sit and stand up from a Bobath table; 6) sit and stand up from a therapy bed; 7) sit and stand up from a chair with wheels; 8) sit and stand up from a chair with handles; 9) walk $3 \mathrm{~m}$, turn around the halfway point, and walk back, holding a cup; and 10) walk $3 \mathrm{~m}$, turn around the halfway point, and walk back, holding a cup of water.

\section{Statistical analysis}

The contents of this study was analyzed, using Version 18.0 of SPSS for Windows. The result values of each experiment were expressed as the average and the standard deviation. The Shapiro-Wilk test to determine the normal distribution of the metrics, but did not meet the normal distribution. A Wilcoxon signed - rank test was conducted to identify before and after change within the group with a nonparametric test. A Mann-Whitney $U$ test was taken to identify changes between groups. The level of statistical significance for measuring items was $\alpha=0.05$.

\section{RESULTS}

\section{General Characteristics of Study Objects}

A total of twelve subjects, 10 men and 2 women, participated in this study. The causes of brain lesions were bleeding and infarction (6 subjects each). Six and six subjects were respectively affected by brain lesions on their left and right side. The average age was 56.33 in the experimental group and 60.33 in the SPO group (Table 1). 


\section{Changes before and after the Experiment between Groups}

As a result of testing the homogeneity between two groups before the experiment, there was no significant difference $(\mathrm{p}>0.05)$. Changes before and after the mediation were analyzed with a Wilcoxon signed-rank test. While it reduced from $18.81 \pm 9.25$ to $16.29 \pm 9.47$ in the AOT group, a change in TUG decreased from $29.91 \pm 22.20$ to $16.91 \pm 7.27$ in the SPO group. Accordingly, there was a significant difference in two groups. On the other hand, there was no significant difference between two groups as a result of comparing STS and DGI before and after the experiment (Table 2).

\section{Changes within Groups}

As a result of testing the homogeneity between two groups before the experiment, there was no significant difference $(p>0.05)$. Changes in UG, STS, and DGI within two groups were analyzed with a Mann-Whitney U test. The AOT group showed a significant difference in TUG, a decrease from $18.81 \pm 9.25$ to $16.29 \pm 9.47$. The SPO group also showed a significant difference, a decrease from $29.91 \pm$ 22.20 to $16.91 \pm 7.27$. However, there was no significant difference in STS and DGI within the group (Table 3).

\section{DISCUSSION}

This study aimed to identify the effects of movement observation

Table 1. General characteristics of the subjects

\begin{tabular}{lcc}
\hline Variable & AOT $_{\text {group }}^{+}(\mathrm{n}=6)$ & SPO group $^{\ddagger}(\mathrm{n}=6)$ \\
\hline Gender & male: 5 & male: 5 \\
female: 1 & female: 1 \\
Lesion side & infarction: 3 & infarction: 3 \\
hesion area & hemorrahge: 3 & hemorrahge : 3 \\
& left: 2 & right: 5 \\
Ages & $56.33 \pm 4.45$ & left: 1 \\
\hline
\end{tabular}

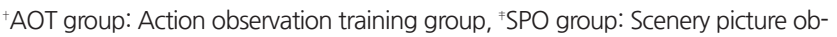
servation. training on chronic stroke patients' TUG movements. It demonstrated that the integration of the movement observation and physical training might improve patients' movement performance..$^{23}$ It is considered to be a functional approach from a clinical respective. Moreover, it may be an alternative which patients can continuously train at home after hospital discharge as a program for patient education and home workout. However, there is no standardized framework to organize functional activities. Therefore, it is required to conduct studies that functional movements are observed and trained, a variety of functional evaluations are made, and specific elements are identified.

This study made a movement observation on the TUG movement, one of the functional activities of chronic stroke patients, based on the mirror neuron system to find changes in the STS movement, the TUG movement, and the dynamic ambulatory capacity before and after the mediation. As a result of the study, there are significant differences in the TUG capability of the AOT group and the SPO group after the mediation. It is assumed that this difference might be caused by the effect of the movement observation. As movement to be trained observed in advance to activate the same movement area, a positive effect was exerted when the movement was actually made. The movement observation training includes a cognitive process as a method to observe a subject's movements, practice them through imitation, and improve relevant movement areas. Moreover, the results of this study correspond

Table 3. The comparison of variances between each group

\begin{tabular}{|c|c|c|c|c|c|}
\hline \multicolumn{2}{|c|}{ Variable } & \multirow{2}{*}{$\begin{array}{c}\text { AOT Group } \\
18.81 \pm 9.25\end{array}$} & \multirow{2}{*}{$\frac{\text { SPO Group }}{29}$} & \multirow{2}{*}{$\frac{z}{-0.801}$} & \multirow{2}{*}{$\frac{p}{-0.730}$} \\
\hline TUG & Pre-test & & & & \\
\hline & Post-test & $16.29 \pm 9.47$ & $16.91 \pm 7.27$ & 0.423 & 0.465 \\
\hline \multirow[t]{2}{*}{ STS } & Pre-test & $13.57 \pm 3.14$ & $13.57 \pm 3.14$ & 0.000 & 0.000 \\
\hline & Post-test & $13.10 \pm 3.41$ & $13.10 \pm 3.41$ & 1.000 & 1.000 \\
\hline \multirow[t]{2}{*}{ DGI } & Pre-test & $18.50 \pm 4.80$ & $13.66 \pm 5.68$ & 0.147 & 0.520 \\
\hline & Post-test & $18.00 \pm 6.19$ & $17.40 \pm 3.78$ & 0.180 & 0.537 \\
\hline
\end{tabular}

${ }^{+}$AOT group: Action observation training group, ${ }^{\ddagger}$ SPO group: Scenery picture observation.

Table 2. The comparison of variances within in each group

\begin{tabular}{|c|c|c|c|c|c|c|c|c|}
\hline \multirow{2}{*}{ Variable } & \multicolumn{4}{|c|}{ AOT Group ${ }^{+}$} & \multicolumn{4}{|c|}{ SPO Group ${ }^{\dagger}$} \\
\hline & pre-test & post-test & z & $p *$ & pre-test & post-test & z & $p$ \\
\hline TUG & $18.81 \pm 9.25$ & $16.29 \pm 9.47$ & -0.80 & $0.42^{*}$ & $29.91 \pm 22.20$ & $16.91 \pm 7.27$ & -0.73 & 0.46 \\
\hline STS & $13.57 \pm 3.14$ & $13.10 \pm 3.41$ & 0.00 & 1.00 & $13.57 \pm 3.14$ & $13.10 \pm 3.41$ & 0.00 & 1.00 \\
\hline DGI & $18.50 \pm 4.80$ & $18.00 \pm 6.19$ & -1.45 & 0.15 & $13.66 \pm 5.68$ & $17.40 \pm 3.78$ & -0.64 & 0.52 \\
\hline
\end{tabular}

${ }^{*} \mathrm{p}<0.05,{ }^{+}$AOT group: Action observation training group, ${ }^{*}$ SPO group: Scenery picture observation. 
with the results of studies on the effects of a movement observation, stating that a movement observation has a positive effect on the imitation and performance of movements. A decrease in the TUG time in two groups is considered a result that subjects repeatedly performed and practiced the same physical training called "TUG". It is shown that there is no difference in STS and DGI in two groups. It is assumed that the movement observation and physical training were focused on TUG, and TUG did not have learning and transition effects on separate tasks, STS and the ambulatory task, as one mobility task.

In movement observation training, it is required to observe, imitate, and practice other people's actions, and mobilize the mirror neuron system areas through the activation of the brain area, which is obtained based on the actual movement experience. In a study for healthy adults, Celnik et al. ${ }^{24}$ found that the AOT group which actually observed similar movements and repeatedly practiced observed movements showed a more significant difference than the training group which repeated simple movements without any observation on movements. Pelosin et al. ${ }^{25}$ maintain that there is a significant difference after a movement observation was made to reduce freezing of gate, shown in patients with Parkinson's disease. His finding is consistent with the result of this study.

This study identified changes in the TUG movement before and after training. Normal adults' performance times for the TUG are below 10 seconds, ${ }^{26}$ while those of normal adults in their sixties are between 8 and 13.1 seconds. ${ }^{27}$ A reduction in the performance time for the TUG means an improvement in joint position sense, plantar flexion muscles, dynamic balance control, and ambulatory capability. $^{28}$ As a result of conducting circulatory task-oriented gait training for twelve chronic stroke patients, it was reported that the performance time reduced from 27.4 before training to 19 seconds after training. Moreover, the experimental group showed 23.6 seconds, a more significant difference than the SPO group, in a post-test made in four weeks. The performance times of the AOT group in this study reduced from $18.81 \pm 9.25$ before the mediation to $16.29 \pm 9.47$ after the mediation, showing a statistically significant difference $(\mathrm{p}>0.05)$. Those of the SPO group reduced from $29.91 \pm 22.20$ before the mediation to $16.91 \pm 7.27$ after the mediation, showing a statistically significant difference $(p>0.05)$. These results are consistent with those of previous studies. ${ }^{23}$ It is assumed that the repeated exercise in the AOT group and the SPO group had a positive effect on learning.
In case of stroke patients, the transfer of momentum after lesions, and the generation of force which occurs before and after have effects on the TUG movement due to a reduction in a driving force to stand up and a braking power to control the position. ${ }^{29}$ In this study, it is shown that subject's TUG scores are lower than those (8-13.1 seconds) of normal adults in their sixties. As a result of the training in this study, the TUG scores are improved in both of the MO and the SPO group. The main reason of this result may be that the movement observation training exerted a positive effect on an improvement in the transfer of momentum, a driving force, and a braking power, the qualitative variables of the TUG movement.

This study examined the effect of movement observation training on stroke patients' TUG movements. Study subjects were divided into two groups, the experimental group supposed to observe movements related to daily life, and the SPO group scheduled to observe scenery pictures unrelated to daily life. As a result of an experiment, there is a significant difference in the TUG movement capability between two groups. These results indicate that movement observation training may influence the TUG movement. However, it was difficult to compare the results of this study with those of other studies because there were a few studies on the movement observation training to identify changes in the TUG movement for chronic stroke patients. It was also difficult to generalize the results of this study because the number of study subjects was small and only the change value of the TUG, a functional evaluation tool, was examined. Moreover, this study has more limits in that it was hard to judge the long-term effects of movement observation training through four week training, and the persistence of its effect is unknown because the process was not evaluated.

This study aimed to identify the effects of movement observation training on chronic stroke patients' TUG movements. It demonstrated that the integration of the movement observation and physical training might improve patients' movement performance. ${ }^{23}$ It is considered to be a functional approach from a clinical respective. Moreover, it may be an alternative which patients can continuously train at home after hospital discharge as a program for patient education and home workout. However, there is no standardized framework to organize functional activities. Therefore, it is required to conduct studies that functional movements are observed and trained, a variety of functional evaluations are made, and specific elements are identified. 


\section{REFERENCES}

1. Sale P, Franceschini M. Action observation and mirror neuron network: A tool for motor stroke rehabilitation. Eur J Phys Rehabil Med. 2012; 48(2):313-8

2. Ryerson TB, Trainer M, Holloway JS et al. Observations of ozone formation in power plant plumes and implications for ozone control strategies. Science. 2001;292(5517):719-23.

3. Bensoussan L, Mesure S, Viton JM et al. Kinematic and kinetic asymmetries in hemiplegic patients' gait initiation patterns. J Rehabil Med. 2006 38(5):287-94.

4. Sharp SA, Brouwer BJ. Isokinetic strength training of the hemiparetic knee: Effects on function and spasticity. Arch Phys Med Rehabil. 1997; 78(11):1231-6.

5. Schenkman M, Berger RA, Riley PO et al. Whole-body movements during rising to standing from sitting. Phys Ther. 1990;70(10):638-48; discussion 48-51.

6. Janssen WG, Bussmann HB, Stam HJ. Determinants of the sit-to-stand movement: A review. Phys Ther. 2002;82(9):866-79.

7. Kerr A, Durward B, Kerr KM. Defining phases for the sit-to-walk movement. Clin Biomech (Bristol, Avon). 2004;19(4):385-90.

8. Magnan A, McFadyen BJ, St-Vincent G. Modification of the sit-to-stand task with the addition of gait initiation. Gait \& Posture. 1996;4(3):232-41.

9. Lee J, Nam K, Kim K et al. Effect of weight bearing exercise on weight bearing and balance for patients with chronic stroke. J Korean Soc Phys Ther. 2012;24:253-61.

10. Brunt D, Vander Linden DW, Behrman AL. The relation between limb loading and control parameters of gait initiation in persons with stroke. Archives of physical medicine and rehabilitation. 1995;76(7):627-34.

11. Hesse S, Schauer M, Malezic M et al. Quantitative analysis of rising from a chair in healthy and hemiparetic subjects. Scand J Rehabil Med. 1994; 26(3):161-6.

12. Engardt M, Olsson E. Body weight-bearing while rising and sitting down in patients with stroke. Scand J Rehabil Med. 1992;24(2):67-74.

13. Malouin F, Richards CL, Doyon J et al. Training mobility tasks after stroke with combined mental and physical practice: A feasibility study. Neurorehabil Neural Repair. 2004;18(2):66-75.

14. Refshauge KM, Ada L, Ellis E. Science-based rehabilitation: Theories into practice. Elsevier Health Sciences, 2005: Pages.

15. Malouin F, McFadyen B, Dion L et al. A fluidity scale for evaluating the motor strategy of the rise-to-walk task after stroke. Clin Rehabil. 2003; 17(6):674-84.

16. Dobkin BH. Training and exercise to drive poststroke recovery. Nat Clin Pract Neurol. 2008;4(2):76-85.

17. Garrison GM, Bernard ME, Rasmussen NH. 21st-century health care: The effect of computer use by physicians on patient satisfaction at a family medicine clinic. Fam Med. 2002;34(5):362-8.

18. Rizzolatti G. The mirror neuron system and its function in humans. Anat Embryol (Berl). 2005;210(5-6):419-21.

19. Fogassi L, Ferrari PF, Gesierich B et al. Parietal lobe: From action organization to intention understanding. Science. 2005;308(5722):662-7.

20. Holmes AM. The cno and the aco: An alphabet soup of healthcare reform. Nurs Manage. 2011;42(8):46-8.

21. Cattaneo L, Rizzolatti G. The mirror neuron system. Arch Neurol. 2009; 66(5):557-60.

22. Iacoboni M. Neural mechanisms of imitation. Curr Opin Neurobiol. 2005;15(6):632-7.

23. Jung HY, Choi JD. The effects of vestibular sensory stimulation training on balance and gait in the patients with stroke. J Korean Soc Phys Ther. 2014;26(5):365-71.

24. Celnik P, Stefan K, Hummel F et al. Encoding a motor memory in the older adult by action observation. Neuroimage. 2006;29(2):677-84.

25. Pelosin E, Avanzino L, Bove M et al. Action observation improves freezing of gait in patients with parkinson's disease. Neurorehabil Neural Repair. 2010;24(8):746-52.

26. Podsiadlo D, Richardson S. The timed "up \& go": A test of basic functional mobility for frail elderly persons. J Am Geriatr Soc. 1991;39(2): $142-8$.

27. Bohannon RW. Reference values for the timed up and go test: A descriptive meta-analysis. J Geriatr Phys Ther. 2006;29(2):64-8.

28. Ng SS, Hui-Chan CW. The timed up \& go test: Its reliability and association with lower-limb impairments and locomotor capacities in people with chronic stroke. Arch Phys Med Rehabil. 2005;86(8):1641-7.

29. Bowden MG, Balasubramanian CK, Neptune RR et al. Anterior-posterior ground reaction forces as a measure of paretic leg contribution in hemiparetic walking. Stroke. 2006;37(3):872-6. 